Artigo Original

Saúde Funcional

\title{
AVALIAÇÃO BAROPODOMÉTRICA E DO EQUILÍBRIO EM PACIENTES COM DISTROFIA MUSCULAR DE STEINERT ANTES E APÓS A PRÁTICA DO WII REABILITAÇÃO
}

\section{Baropodometric and balance evaluation in patients with Steinert muscular dystrophy before and after the practice with WII rehabilitation}

Gabriela Casagrande Jeremias ${ }^{1}$, Camila Ferraz ${ }^{1}$, Évelin Vicente ${ }^{2}$

\footnotetext{
${ }^{1}$ Acadêmica do Curso de Fisioterapia da Universidade do Extremo Sul Catarinense.

${ }^{2}$ Docente do Curso de Fisioterapia da Universidade do Extremo Sul Catarinense.
}

Endereço para correspondência:

*Évelin Vicente

Universidade do Extremo Sul Catarinense, Curso de Fisioterapia

Avenida Universitária, 1105 - Bairro Universitário

CEP: 88806-000 - Criciúma-SC, Brasil.

Email: eve@unesc.net 


\section{Artigo Original}

\section{Saúde Funcional}

\section{Resumo}

As Distrofias Musculares são miopatias de caráter crônico e progressivo dentre elas pode-se destacar a Distrofia Muscular de Steinert (DMS) que apresenta alteração de equilíbrio. O objetivo deste estudo foi avaliar os efeitos do Wii reabilitação sobre o equilíbrio no paciente com distrofia muscular por meio da baropodometria. A amostra foi composta por 2 participantes do sexo masculino, de 17 e 18 anos, com Distrofia Muscular de Steinert. Um paciente foi o controle, fazendo a fisioterapia convencional e não praticando o Wii, o outro paciente fez a fisioterapia convencional e realizou a prática do Wii. O equilíbrio estático e dinâmico foi avaliado pelo Baropodômetro e pela escala de Equilíbrio de Berg (EEB), o controle de tronco foi avaliado pela Escala de Comprometimento de Tronco (ECT). De acordo com a EEB e a ECT o paciente Wii obteve maior controle de tronco e melhora do equilíbrio. Na avaliação baropodométrica estática e dinâmica o indivíduo praticante do Wii obteve melhora geral através dos itens analisados, em relação ao paciente que realizou a fisioterapia convencional. O presente estudo demonstra que a fisioterapia associada ao treino de equilíbrio com Nintendo Wii apresenta resultados satisfatórios na reabilitação dos pacientes com DMS.

Palavras-chaves: Distrofias Musculares, Equilíbrio Postural, Fisioterapia.

\section{Abstract}

Muscular dystrophies are chronic and progressive myopathies. Among them, we can highlight the Muscular Dystrophy Steinert (DMS) that presents the alteration in the balance. The aim of this study was evaluate the effects of Wii rehabilitation in the balance of patients with muscular dystrophy by baropodometry. The sample consisted of two male participants, 17 and 18 years old, with Muscular Dystrophy Steinert. One patient was the control, making conventional physiotherapy and not practicing the Wii. The other one received conventional physical therapy and practiced Wii. The static and dynamic balance was evaluated using the Baropodometry and Berg Balance Scale (BBS). The Trunk Control Test (TCT) was evaluated using the Trunk Impairment Scale. According to the BBS and the TCT, the patient that used Wii obtained greater trunk control and improved the balance. In the static and dynamic baropodometric evaluation, the practitioner of Wii obtained general improvement through the items analyzed compared to the patient who practiced conventional physical therapy. The present study 


\section{Artigo Original}

\section{Saúde Funcional}

demonstrates that physical therapy associated with balance training on Nintendo Wii presents satisfactory results in the rehabilitation of patients with DMS.

Keywords: Muscular Dystrophies, Balance Postural, Physical Therapy.

\section{INTRODUÇÃO}

As distrofias musculares são miopatias, de caráter hereditário, comprometimento progressivo e irreversível da musculatura esquelética. Sua classificação ocorre de acordo com a forma de transmissão hereditária1.

O diagnóstico da distrofia muscular se baseia nas manifestações clínicas e nos antecedentes familiares, sendo confirmado pela biopsia muscular, análise e estudo do DNA e pela eletromiografia ${ }^{2}$.

Entre as diferentes formas de distrofia muscular encontra-se a Distrofia Miotônica de Steinert, a qual atinge ambos os sexos, sendo a miopatia degenerativa mais comum em adultos. Aparece progressivamente mais cedo em gerações sucessivas, acompanhada do aumento de sua severidade ${ }^{3}$. O quadro clínico mais frequente consiste em transtornos musculares em forma de miotonia, atrofia e desgaste progressivo da musculatura. A doença evolui entre quinze e vinte anos, a partir do início dos sintomas, época em que ocorre impossibilidade para marcha e grave diminuição no grau de independência motora ${ }^{4}$.

O equilíbrio é a integração sensória motora que garante a manutenção da postura corporal, sendo um trabalho integrado e simultâneo dos sistemas nervoso, sensorial e motor. O sistema sensorial fornece o posicionamento do corpo em relação ao ambiente e a outros segmentos, enquanto o sistema motor ativa os músculos para realização do movimento. O Sistema nervoso central (SNC) conecta as informações advindas do sistema sensorial para enviar impulsos nervosos aos músculos. Qualquer 


\section{Artigo Original}

\section{Saúde Funcional}

falha em um desses sistemas pode causar desequilíbrio ${ }^{5}$.

A baropodometria trata-se de um recurso utilizado para avaliar e mensurar a distribuição da pressão plantar, em situações estáticas e dinâmicas, bem como, a realização da estabilometria. Esta por sua vez, é um método de análise do equilíbrio postural por meio das quantificações das oscilações do corpo, sendo um excelente método para ser usado na avaliação clínica ${ }^{5}$.

Pesquisas recentes têm utilizado os jogos do Nintendo® Wii para verificar seus possíveis benefícios no processo de reabilitação, adicionando um fator motivacional na sessão fisioterapêutica ${ }^{6}$. Dentre os benefícios do Wii reabilitação podese destacar as correções posturais e de equilíbrio, assim como o aumento da capacidade de locomoção ${ }^{7}$.

Diante disso, o presente estudo teve como objetivo avaliar os efeitos do Wii reabilitação sobre o equilíbrio no paciente com distrofia muscular da ASCADIM por meio da baropodometria.

\section{METODOLOGIA}

A pesquisa foi aprovada pelo Comitê de Ética em Pesquisa da Universidade do Extremo Sul Catarinense (UNESC) sob o protocolo de ㄲo 729.668/2014. O estudo foi realizado na Associação Sul Catarinense de Amigos e Familiares de Portadores de Distrofia Muscular Progressiva (ASCADIM) em Criciúma, Brasil.

A amostra foi composta por dois pacientes da ASCADIM, sendo estes do sexo masculino, com idade entre 17 e 18 anos, apresentando Distrofia Miotônica de Steinert. Aleatoriamente um paciente foi escolhido como controle, fazendo apenas a Fisioterapia convencional. $O$ outro paciente além de receber a Fisioterapia convencional, recebeu a prática do Wii. 


\section{Artigo Original}

\section{Saúde Funcional}

Como critérios de inclusão, estão os pacientes membros da ASCADIM com diagnóstico clínico de Distrofia Miotônica de Steinert, que permanecem em posição ortostática sem apoio, e aceitaram participar da pesquisa assinando o Termo de Consentimento Livre e Esclarecido (TCLE). O paciente com menos de 18 anos teve o TCLE assinado pelos seus responsáveis. Foram excluídos da pesquisa pacientes que não conseguirem ficar em posição ortostática, que não tinham a Distrofia Miotônica de Steinert, que apresentavam déficit cognitivo e/ou não aceitaram participar da pesquisa.

Ao início do estudo os pacientes foram avaliados. As sessões de terapia convencional dos pacientes ocorreram 2 vezes por semana. A prática do Wii ocorreu 1 vez na semana. Todas as sessões, tanto a Fisioterapia convencional como a prática do Wii, tiveram a duração de 30 minutos. Sendo realizada no período de agosto a outubro de 2014, num total de 10 sessões. Ao final das 10 sessões os pacientes foram reavaliados.

Para avaliação do equilíbrio estático e dinâmico foi aplicada a Escala de Equilíbrio de Berg composta por 14 tarefas funcionais gerando no final uma pontuação ${ }^{8}$.

O controle de tronco foi verificado através da escala de comprometimento do tronco, que é composta por sete itens. $O$ escore para cada item varia de 0 a 3 e o melhor resultado corresponde à pontuação total de $21^{9}$.

A avaliação da distribuição do peso da planta dos pés foi realizada através do Baropodômetro S-Plate (Estabilometria). Marca Medicapteurs $\AA_{\text {. Plataforma com } 610}$ $\mathrm{mm}$ largura, $580 \mathrm{~mm}$ profundidade, $4 \mathrm{~mm}$ espessura, pesando $6,5 \mathrm{~kg}$. Área equipada com 1600 sensores pizoelétricos de pressão (48x48), tendo150 imagens por segundo de frequência de aquisição. Na avaliação baropodométrica estática, os pacientes permaneceram em posição ortostática sobre a plataforma, com apoio bipodálico, braços ao longo do corpo, durante 30 segundos com os olhos abertos e, em seguida, com os olhos fechados. Na avaliação baropodométrica dinâmica, os pacientes caminharam sobre a plataforma, completando uma passada de locomoção. As análises 


\section{Artigo Original}

\section{Saúde Funcional}

baropodométricas (estática e dinâmica) foram realizadas sem calçados. As coletas dos dados foram realizadas antes e após a intervenção ${ }^{10}$.

Para aplicação do treino de equilíbrio foi utilizado como forma de intervenção, o vídeo game,da marca Nintendo®, denominado Wii, atribuindo a este, o jogo Wii Fit. Para o presente estudo foram utilizados seis jogos específicos para equilíbrio que fizeram parte do protocolo de atendimento ${ }^{11}$.

Durante as sessões com o Nintendo® Wii juntamente com a plataforma Balance Board foram utilizados 6 tipos de exercícios, sendo eles: TableTilt, Penguin Slide, Balance Bubble, Ski slalom, Perfect 10, TiltCity.Cada exercício teve um intervalo de tempo para o descanso de acordo com o nível de condicionamento do paciente.

Os dados foram analisados no programa Microsoft Excel versão 2007. Os resultados oriundos das Escalas de Comprometimento de Tronco e Escala de Equilíbrio de Berg foram expressas em números absolutos. Os resultados da Baropodometria estática e dinâmica foram expressas por meio de média e desvio padrão. A sistematização dos dados foi realizada por meio de tabelas e gráficos.

\section{RESULTADOS}

A Figura 01 mostra os valores da análise de equilíbrio de acordo com a pontuação da Escala de Equilíbrio de Berg, comparando o paciente controle e o paciente Wii, antes e após a intervenção. Na primeira avaliação, antes da intervenção, o paciente controle obteve 47 pontos, e regrediu para 42 pontos após as sessões de fisioterapia. Já na primeira avaliação, antes da intervenção, o paciente Wii obteve 51 pontos, e atingiu 52 pontos a pós a intervenção. 


\section{Artigo Original}

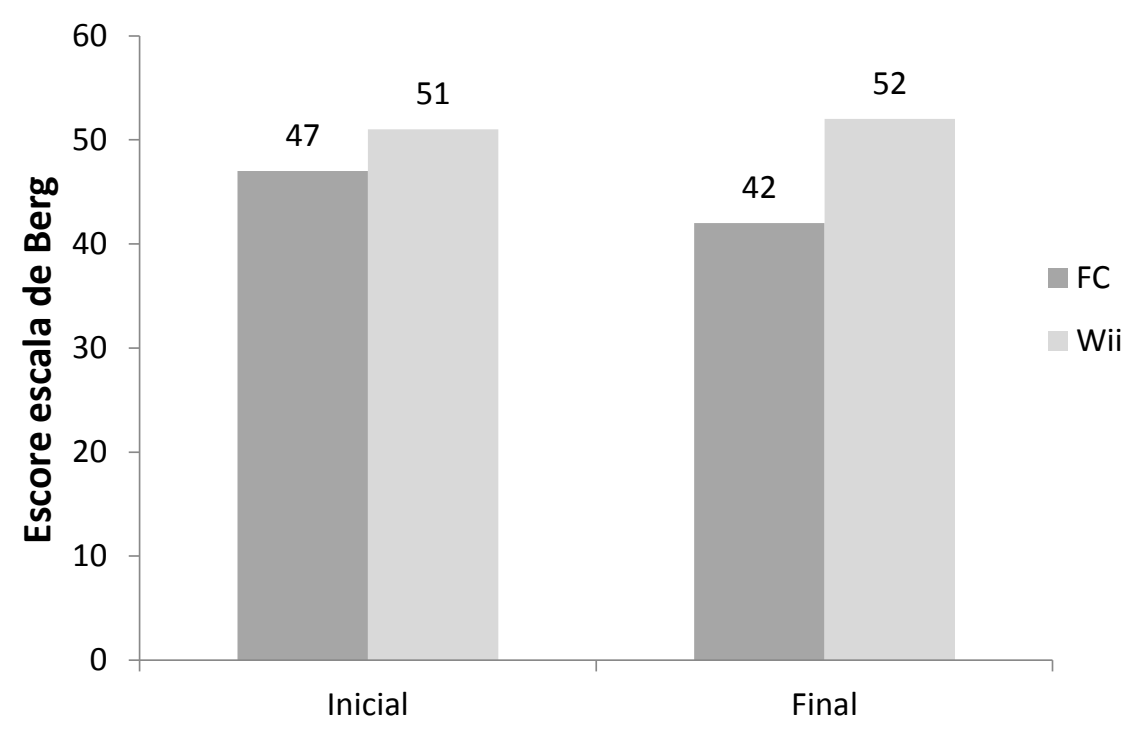

Figura 01. Resultado da Escala de Equilíbrio de Berg do paciente que realizou a Fisioterapia Convencional (FC) e do Paciente Wii, ao término das 10 sessões os pacientes foram reavaliados.

A figura 02 mostra os valores da análise do controle de tronco de acordo com a pontuação da Escala de Comprometimento de tronco, comparando o paciente controle e o paciente Wii, antes e após a intervenção. Na primeira avaliação, antes da intervenção, o paciente controle obteve 17 pontos, e regrediu para 12 pontos após as sessões de fisioterapia. Já na primeira avaliação, antes da intervenção, o paciente Wii obteve 14 pontos, e atingiu 16 pontos a pós a intervenção. 


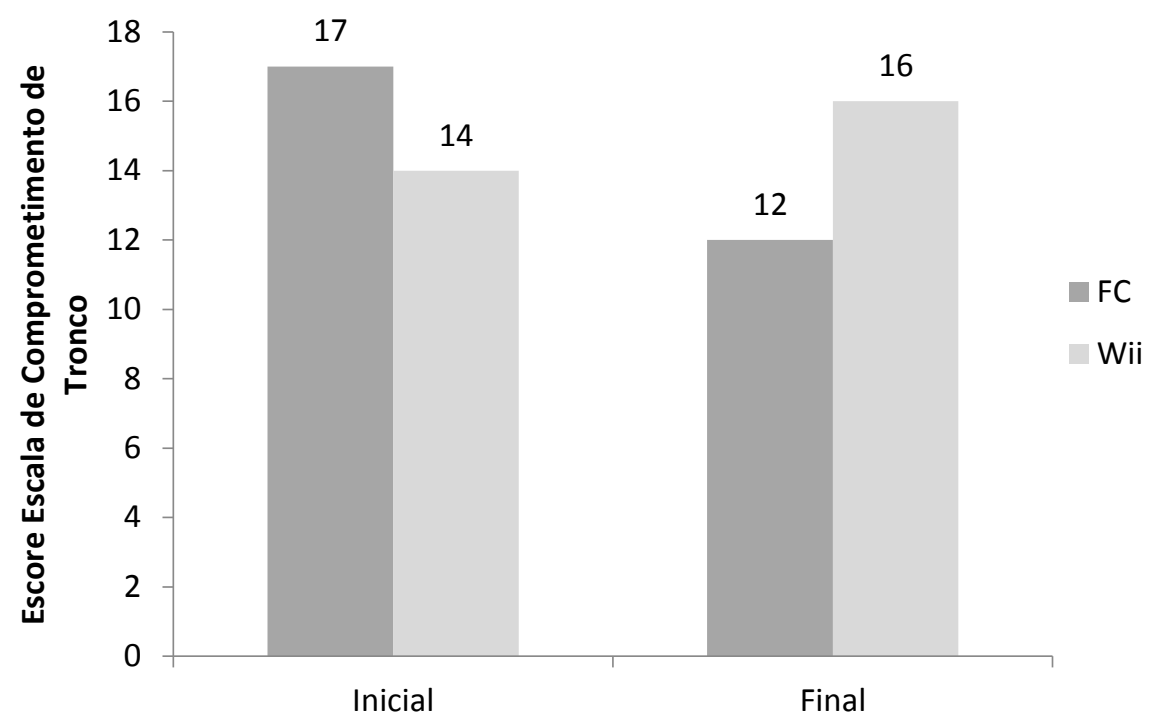

Figura 02. Resultado da Escala de Comprometimento de Tronco do paciente que realizou a Fisioterapia Convencional (FC) e do Paciente Wii, ao término das 10 sessões os pacientes foram reavaliados.

A Tabela 01 mostra os resultados obtidos na baropodometria estática, onde se pode observar que no item Pressão Direito e Esquerdo (D/E) obteve-se uma melhora tanto de Olhos Abertos (OA), quanto de Olhos Fechados (OF), no paciente Wii. No Pico Antepé $\mathrm{E}$ houve uma melhora somente de OF no paciente Wii, assim como no paciente controle. No Pico do Antepé e Retropé D, pode-se observar uma melhora nos OA e OF no paciente Wii. No item Comprimento, no paciente Wii, houve uma melhora somente nos OF, já no paciente controle obteve-se melhora tanto de OA quanto de OF. Na Área, obteve-se uma melhora nos OA e OF tanto no paciente Wii quanto no paciente controle. $\mathrm{Na}$ largura L/L, obteve-se melhora somente nos OF no paciente controle e na larguraA/P houve melhora nos OF do paciente Wii e nos OA e OF no paciente controle. Nos itens Desvio Médio Látero Lateral (L/L) e Ântero Posterior (AP) houve uma melhora nos OA e OF no paciente Wii. Na Velocidade Média L/L, obteve-se melhora nos OA e OF no paciente Wii, contudo, na Velocidade Média A/P houve melhora somente nos OA do paciente Wii. 


\section{Artigo Original}

\section{Saúde Funcional}

Tabela 01. Resultado da Baropodometria Estática com os Olhos Abertos (OA) e Olhos

Fechados (OF) do paciente que realizou a Fisioterapia Convencional (FC) e do Paciente Wii, ao término das 10 sessões os pacientes foram reavaliados.

\begin{tabular}{|c|c|c|c|c|c|}
\hline \multirow{2}{*}{\multicolumn{2}{|c|}{ Variáveis }} & \multicolumn{2}{|c|}{ Wiiterapia } & \multicolumn{2}{|c|}{ Fisioterapia Convencional } \\
\hline & & $\mathrm{AO}$ & OF & $\mathrm{AO}$ & OF \\
\hline \multirow{2}{*}{$\begin{array}{c}\text { Pressão D/E } \\
(\%)\end{array}$} & Antes & $5,20 \pm 3,14$ & $4,20 \pm 4,10$ & $12,41 \pm 11,71$ & $12,38 \pm 10,81$ \\
\hline & Depois & $6,33 \pm 6,10$ & $4,77 \pm 7,19$ & $8,00 \pm 5,42$ & $9,41 \pm 6,99$ \\
\hline \multirow{2}{*}{$\begin{array}{c}\text { Picos Antepé } \\
E\left(a / \mathrm{cm}^{2}\right)\end{array}$} & Antes & $639,3 \pm 121,5$ & $608,1 \pm 112,4$ & $622,4 \pm 83,52$ & $559,61 \pm 103,5$ \\
\hline & Depois & $615,5 \pm 122,6$ & $611,1 \pm 108,7$ & $611,9 \pm 165,2$ & $611,36 \pm 94,00$ \\
\hline \multirow{2}{*}{$\begin{array}{c}\text { Picos Retropé } \\
E\left(a / \mathrm{cm}^{2}\right)\end{array}$} & Antes & $904,1 \pm 222,6$ & $816,5 \pm 131,3$ & $815,4 \pm 176,4$ & $781,2 \pm 145,7$ \\
\hline & Depois & $933,6 \pm 144,2$ & $861,4 \pm 111,1$ & $945,4 \pm 144,3$ & $867,4 \pm 122,7$ \\
\hline \multirow{2}{*}{$\begin{array}{c}\text { Picos Antepé } \\
D\left(\mathrm{a} / \mathrm{cm}^{2}\right)\end{array}$} & Antes & $496,6 \pm 141,8$ & $485,1 \pm 141,1$ & $600,5 \pm 110,5$ & $565,5 \pm 167,4$ \\
\hline & Depois & $700,2 \pm 198,4$ & $639,8 \pm 166,2$ & $538,8 \pm 100,0$ & $554,63 \pm 90,92$ \\
\hline \multirow{2}{*}{$\begin{array}{c}\text { Picos Retropé } \\
D \\
\left(\mathrm{a} / \mathrm{cm}^{2}\right) \\
\end{array}$} & Antes & $962,8 \pm 330,9$ & $852,5 \pm 145,9$ & $761,6 \pm 185,4$ & $774,76 \pm 160,9$ \\
\hline & Depois & $896,6 \pm 173,3$ & $800,9 \pm 155,4$ & $930,3 \pm 188,2$ & $777,77 \pm 180,3$ \\
\hline \multirow{2}{*}{$\begin{array}{l}\text { Comprimento } \\
(\mathrm{mm})\end{array}$} & Antes & $50,22 \pm 19,77$ & $42,78 \pm 16,91$ & $38,21 \pm 11,60$ & $42,32 \pm 9,81$ \\
\hline & Depois & $42,46 \pm 7,97$ & $45,66 \pm 12,05$ & $55,26 \pm 23,66$ & $54,68 \pm 22,09$ \\
\hline \multirow{2}{*}{$\begin{array}{l}\text { Área } \\
\left(\mathrm{mm}^{2}\right)\end{array}$} & Antes & $15,24 \pm 25,36$ & $15,64 \pm 12,51$ & $18,66 \pm 16,61$ & $12,45 \pm 23,92$ \\
\hline & Depois & $21,62 \pm 23,66$ & $15,78 \pm 22,99$ & $47,55 \pm 66,31$ & $45,23 \pm 71,19$ \\
\hline \multirow{2}{*}{$\begin{array}{l}\text { Largura L/L } \\
\quad(\mathrm{mm})\end{array}$} & Antes & $5,66 \pm 2,67$ & $4,85 \pm 2,64$ & $5,50 \pm 1,99$ & $34,51 \pm 1,98$ \\
\hline & Depois & $2,48 \pm 4,02$ & $3,03 \pm 1,04$ & $5,44 \pm 2,67$ & $54,62 \pm 3,55$ \\
\hline \multirow{2}{*}{$\begin{array}{l}\text { Largura A/P } \\
\qquad(\mathrm{mm})\end{array}$} & Antes & $7,41 \pm 3,33$ & $5,41 \pm 1,23$ & $4,00 \pm 3,85$ & $4,43 \pm 1,90$ \\
\hline & Depois & $4,89 \pm 2,50$ & $6,11 \pm 3,10$ & $6,81 \pm 7,10$ & $5,66 \pm 5,11$ \\
\hline \multirow{2}{*}{$\begin{array}{l}\text { Desvio Médio } \\
\text { L/L (mm) }\end{array}$} & Antes & $1,00 \pm 0,61$ & $0,83 \pm 0,42$ & $1,13 \pm 0,44$ & $0,06 \pm 0,22$ \\
\hline & Depois & $0,87 \pm 0,72$ & $0,71 \pm 0,41$ & $1,42 \pm 0,81$ & $1,11 \pm 0,96$ \\
\hline \multicolumn{2}{|l|}{ Desvio Médio } & $1,55 \pm 1,22$ & $1,21 \pm 0,24$ & $1,13 \pm 0,44$ & $0,08 \pm 0,54$ \\
\hline
\end{tabular}




\section{Artigo Original}

\section{Saúde Funcional}

\begin{tabular}{cc|c|c|c|c}
\hline A/P $(\mathrm{mm})$ & Depois & $1,12 \pm 0,71$ & $1,11 \pm 0,62$ & $1,72 \pm 1,95$ & $1,15 \pm 1,88$ \\
\hline \multirow{2}{*}{$\begin{array}{c}\text { Velocidade } \\
\text { Média L/L } \\
(\mathrm{mm} / \mathrm{s})\end{array}$} & Antes & $0,45 \pm 0,14$ & $0,94 \pm 0,28$ & $0,25 \pm 0,05$ & $00,82 \pm 0,21$ \\
\cline { 2 - 6 } & Depois & $0,15 \pm 0,04$ & $0,94 \pm 0,25$ & $0,42 \pm 0,10$ & $11,12 \pm 0,42$ \\
\hline $\begin{array}{c}\text { Velocidade } \\
\text { Média A/P } \\
(\mathrm{mm} / \mathrm{s})\end{array}$ & Antes & $0,98 \pm 0,33$ & $0,99 \pm 0,25$ & $0,15 \pm 0,07$ & $0,82 \pm 0,21$ \\
\cline { 2 - 6 } & Depois & $0,81 \pm 0,16$ & $1,11 \pm 0,31$ & $1,3 \pm 0,62$ & $1,08 \pm 0,61$ \\
\hline
\end{tabular}

Valores expressados com média \pm erro padrão (3 avaliações). Exceto para os valores de pressão D/E, os quais são expressos em \%.

A Tabela 02 mostra os resultados obtidos na baropodometria dinâmica, onde se pode observar uma melhora na Área somente no pé direito do paciente Wii. Nos Picos Máximos e Médios, obteve-se uma melhora tanto no pé direito quanto no pé esquerdo do paciente Wii.

Tabela 02. Resultado da Baropodometria Dinâmica do paciente que realizou a Fisioterapia Convencional (FC) e do Paciente Wii, ao término das 10 sessões os pacientes foram reavaliados.

\begin{tabular}{cc|c|c|c|c}
\hline \multirow{2}{*}{ Variáveis } & \multicolumn{2}{c}{ Wiiterapia } & Pisioterapia Convencional \\
\cline { 3 - 6 } & & Pé direito & Pé esquerdo & Pé direito & Pé esquerdo \\
\hline \multirow{2}{*}{$\begin{array}{c}\text { Área } \\
\left(\mathrm{cm}^{2}\right)\end{array}$} & Depois & 96 & 84 & 107 & 121 \\
\hline \multirow{2}{*}{$\begin{array}{c}\text { Picos } \\
\text { máximos } \\
\left(\mathrm{g} / \mathrm{cm}^{2}\right)\end{array}$} & Antes & 1880 & 2906 & 105 & 103 \\
\hline \multirow{2}{*}{$\begin{array}{c}\text { Picos médios } \\
\left(\mathrm{a} / \mathrm{cm}^{2}\right)\end{array}$} & Depois & 1572 & 2505 & 3638 & 2081 \\
\cline { 2 - 6 } & Antes & 921 & 1077 & 597 & 493 \\
\hline
\end{tabular}

Valores expressados em unidades de medida de uma única avaliação.

$\mathrm{Na}$ análise dinâmica, podemos destacar o tipo de pisada do paciente controle e do paciente Wii, conforme observado na Figura 03, onde há predomínio do pé tipo 


\section{Artigo Original}

\section{Saúde Funcional}

cavo e após a intervenção houve uma melhora de distribuição do peso na planta dos pés tendo uma normalização da pisada em relação ao início da intervenção.

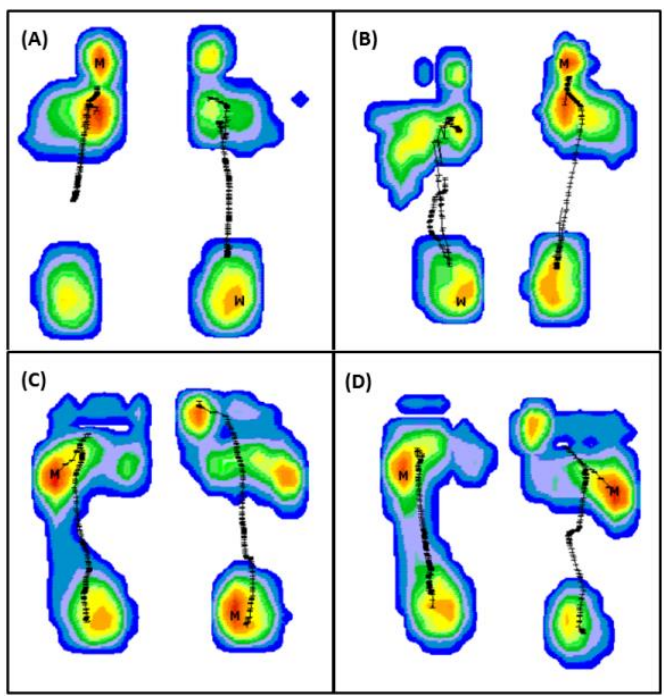

Figura 03. Resultado da avaliação baropodométrica dinâmica dos pacientes que realizaram a Fisioterapia Convencional (FC) e do Paciente Wii, ao término das 10 sessões os pacientes foram reavaliados. (A) Avaliação do paciente Wii, (B) Reavaliação do paciente Wii, (C) Avaliação do paciente controle, (D) Reavaliação do paciente controle.

\section{DISCUSSÃO}

Os sistemas de realidade virtual têm características próprias e de relevante importância no processo de reabilitação, das quais se destacam o fornecimento imediato de feedback e o fato de serem normalmente mais atrativos que os métodos tradicionais, pois são realizados de forma lúdica, podendo ser utilizada como uma forma de terapia complementar. Pode-se destacar o Nintendo Wii que trata-se de um recurso terapêutico que pode ser utilizado como instrumento de reabilitação, tanto nos aspectos motores, como no controle postural e equilíbrio ${ }^{12}$.

O protocolo de tratamento fisioterapêutico proposto mostrou que o paciente obteve melhora no equilíbrio através da Escala de Equilíbrio de Berg (EEB), podendo observar uma melhora da pontuação no equilíbrio do paciente selecionado para este 


\section{Artigo Original}

\section{Saúde Funcional}

estudo. Os ganhos obtidos pelo paciente nesta escala sugerem melhora dos ajustes posturais ${ }^{13}$.

Os resultados do presente estudo mostram que o Nintendo Wiimanteve um nível elevado do escore total do paciente Wii pela EEB. Esses resultados devem ser entendidos pelo fato do paciente Wii já apresentar no início da pesquisa uma boa pontuação. Contudo, a manutenção desses valores após a intervenção realça a associação positiva do Nintendo Wii. A manutenção ou melhora do equilíbrio em pacientes submetidos a programas de treinamento com o Nintendo Wii, deve-se a instituição de oscilações corporais em diversos planos de movimento, de maneira dinâmica e articulada com feedback visual e sonoro, as quais provavelmente estimularam inúmeros proprioceptores ${ }^{14}$.

O protocolo de tratamento fisioterapêutico proposto mostrou que o paciente obteve melhora no controle de tronco através da Escala de Comprometimento de Tronco (ECT). O controle de tronco é uma habilidade motora básica que exerce papel fundamental nas atividades funcionais, proporcionando ao mesmo tempo estabilidade e mobilidade, permitindo ao indivíduo realizar atividades manuais e deambular ${ }^{15}$.

De acordo com os resultados obtidos, na análise baropodométrica, as diferenças significativas encontradas na VM L/L e A/P entre as condições de OA e OF, indicam que quanto maior a velocidade desse deslocamento mais difícil é a manutenção do equilíbrio, ou seja, é importante que os dados referentes à velocidade diminuam. Nos OF da VM A/P, não foi observado melhora pelo fato da retirada do sistema visual promover oscilações no corpo para controle do equilíbrio ${ }^{16}$.

A baropodometria mostrou aumento na área da superfície da região plantar, maior descarga de peso para a região do ante-pé e menor descarga em região do retropé facilitando a manutenção do equilíbrio, principalmente no pé direito, já que uma melhor descarga de peso nos pés indica uma melhora na distribuição da pressão plantar em posição estática ${ }^{17}$.

Em relação ao Desvio Médio (DM) A/P e L/L, houve uma diminuição, sendo um aspecto positivo para os resultados, pois segundo a literatura indivíduos com 


\section{Artigo Original}

\section{Saúde Funcional}

desvios amplos do centro de pressão apresentam um déficit de equilíbrio, a base deste conceito está na ideia de quea redução dasinformações sensoriais contribuem para a falta de controle postural ${ }^{18}$.

Não existem estudos de melhora do equilíbrio em pacientes com Distrofia Muscular de Steinert após treinamento com o programa Nintendo Wii, porém já foram realizados estudos com a mesma finalidade para outras patologias. Um estudo realizado com 13 pacientes com Distrofia Muscular de Duchene (DMD) e a 57 voluntários saudáveis que deslocassem seu centro de gravidade sobre uma plataforma de força fixa, mantendo a posição dos pés. Os autores encontraram que os indivíduos com DMD apresentam a habilidade de movimentar seu centro de gravidade diminuída, em relação a população de meninos saudáveis, sendo que essa habilidade diminuía ainda mais nos estágios próximo a perda da marcha ${ }^{19}$.

Em estudo baropodométrico aplicado em 29 idosos para verificar os efeitos de um programa regular de intervenção fisioterapêutica sobre a distribuição da pressão plantar, obteve-se aumento da área de contato na superfície plantar na posição bipodal após a intervenção, sugerindo facilitação do controle dos movimentos da estabilidade postural, uma vez que o numero de receptores sensoriais da superfície plantar em contato com a superfície também aumenta, suprindo o sistema nervoso com informações mais precisas da periferia, podendo tornar os indivíduos menos propensos a quedas ${ }^{20}$.

Estudo com pacientes hemiparéticos observou-se melhora no equilíbrio utilizando-se a fisioterapia convencional apenas e a fisioterapia convencional associada ao treino com o Nintendo Wii. Um destaque com relação ao grupo que foi submetido ao treino com Wii foi à diminuição na oscilação $L / L$ e A/P de olhos abertos e fechados, não observado no grupo que utilizou apenas a fisioterapia convencional ${ }^{21}$. Tal melhora pode ser atribuída ao fato da Wii reabilitação proporcionar uma maior interação entre os sistemas responsáveis pelo controle postural, trabalhando deslocamentos, feedback visual, estímulos proprioceptivos e auditivos, que repercutem diretamente no aperfeiçoamento da estabilidade postural ${ }^{22}$. 


\section{Artigo Original}

\section{Saúde Funcional}

Os resultados obtidos no presente estudo sugerem que o treino de equilíbrio associado ao programa Wii Fit, proporciona bons resultados na reabilitação fisioterapêutica. Contudo, a fisioterapia convencional mantém sua importância, pois o paciente submetido a este tipo de tratamento, também obteve resultados significantes na reabilitação ${ }^{21}$.

O pé humano constitui a base de apoio e propulsão para a marcha, além de fornecer suporte e flexibilidade para uma transferência e sustentação de peso adequada. Uma adequada biomecânica do pé é responsável pela manutenção da postura e uma distribuição simétrica da pressão plantar, além de exercer um efeito importante no controle postural durante a posição ortostática e na marcha ${ }^{23}$.

Levando em consideração a pisada dos pacientes participantes do presente estudo, os mesmos apresentavam um pé do tipo cavo, que se caracteriza por um aumento do arco plantar, o que significa uma redução na área da planta dos pés que é utilizada para o apoio ${ }^{24}$.

Estudos relatam que indivíduos com um arco plantar normal apresentam melhor equilíbrio postural que aqueles que possuem pés cavos ou planos. A diminuição na área de contato plantar gera um menor bloqueio anatômico entre o aspecto medial do pé e a plataforma de apoio, diminuindo assim a informação sensorial plantar ${ }^{25}$.

Outros autores também colocam que pés cavos recebem menos entradas aferentes dos receptores cutâneos, os quais podem ter mecanismos de controle menos eficientes na sua postura em pé durante o apoio ${ }^{25}$.

De acordo com os resultados apresentados pela análise da baropodometria dinâmica pode ser evidenciado que houve aumento da área de contato plantar e melhor distribuição de apoio entre os pés devido à diminuição dos picos médio e máximo, notando-se uma tendência a melhor distribuição no apoio plantar no paciente praticante do Nintendo $\mathrm{Wi}^{26}$.

Em estudo realizado com 11 indivíduos que teve como objetivo realizar um comparativo das analises dos tipos de pés antes e após os exercícios de força e treino de equilibro, observou-se que após os exercícios propostos houve um aumento na 


\section{Artigo Original}

\section{Saúde Funcional}

sobrecarga no quadrante posterior direito. Ocorreu um aumento na superfície de contato dos pés, evidenciado mais no lado esquerdo, e uma melhor distribuição do apoio plantar entre os pés, melhorando a base de sustentação, dando melhor estabilidade, distribuindo melhor o peso, aumentando o apoio entre os pés e a superfície de contato ${ }^{26}$.

\section{CONCLUSÃO}

Considerando que a DMS é caracterizada como uma patologia de caráter crônico e progressivo torna-se importante a manutenção do quadro clínico do paciente, visto que não há grandes chances de evolução do paciente. Contudo estabilizar seu desempenho relacionado ao equilíbrio é de grande valia para a manutenção da posição ortostática prevenindo possíveis quedas e auxiliando na marcha.

A melhora do equilíbrio não foi comprovada estatisticamente, mas sugere-se a realização de estudo com maior rigor metodológico que avaliem especificamente os aspectos pertinentes a cada patologia neurológica e sua associação aos procedimentos de intervenção do Wii Reabilitação, tais como: duração da intervenção, número de sessões, frequência e tipos de jogos para que haja padronização de protocolos de tratamento, a fim de garantir uma maior confiabilidade e aplicabilidade do recurso junto à fisioterapia neurológica.

Por se tratar de um estudo com somente dois participantes, fazem-se necessárias novas pesquisas com maior número de indivíduos utilizando- se os mesmos jogos virtuais e idênticas ferramentas de mensuração, durante um período similar de intervenção, a fim de que seja possível realizar a comparação dos dados adquiridos na atual pesquisa.

Percebe-se, ainda, que a literatura não é vasta. Há carência de estudos científicos acerca do atendimento específico ao portador de DMS, e que comprovem a eficiência das técnicas utilizadas no tratamento fisioterapêutico. 


\section{Artigo Original}

\section{Saúde Funcional}

\section{REFERÊNCIAS}

1. Rowland LP. Merritt tratado de neurologia. 11. ed. Rio de Janeiro: Guanabara Koogan, 2007.

2. Shepherd RB. Fisioterapia em pediatria. 3ed. São Paulo: Santos Ed., 1998.421 p.

3. Zanoteli E. Ressonância Magnética dos músculos da mastigação e da articulação temporomandibular na Distrofia de Steinert (Tese) São Paulo: UNIFESP, 2000, 77P.

4. Carenzi T, Cunha MB. Abordagem Hidroterapêutica em fortalecimento muscular para pacientes portadores de distrofia miotônica de Steinert: Uma revisão bibliográfica. ConScientiaeSaúde 2003;2:83-8.

5. Rubira APFA, Martins MSE, Dentf CBS, Gerlin NG, Tomaz C, Rubira MC. Eficiência da estabilometria e baropodometria estática na avaliação do equilíbrio em pacientes vestibulopatas. Revista Neurobiologia 2010;72(2):57-64.

6. Dias RS, Sampaio ILA, Taddeo LS. Fisioterapia $x$ Wii: a introdução do lúdico no processo de Reabilitação de pacientes em tratamento fisioterápico. Simpósio Brasileiro de Jogos e Entretenimento Digital, 8th- 10th, Rio de Janeiro, 2009, 34-37.

7. Merians AS, Jack D, Boian R, Tremaine M, Burdea GC, Adamovich SV, Recce M, Poizner H. Virtual Reality-Augmented Rehabilitation for Patients Following Stroke. Physical Therapy 2002;82(9):898-15.

8. Silva A, Almeida G, Cassilhas R, Cohen M, Peccin MS, Tufik S, De Mello MT. Equilíbrio, Coordenação e Agilidade de Idosos Submetidos à Prática de Exercícios Físicos Resistidos. Revista Brasileira Medicina do Esporte 2008;14(2):88-93.

9. Lima NMFV, Rodrigues SY, Fillipo TM, De Oliveira R, Oberg TD, Cacho EWA. Versão brasileira da Escala de comprometimento do Tronco: um estudo de validade em sujeitos pós-acidente vascular encefálico. Fisioterapia e Pesquisa2008; 15(3):248-53.

10. Tafner BN. Análise estabilométrica da influência da oclusão na postura ortostática. Artigos de periódico (graduação). Blumenau: URB, 2010, 53p. 


\section{Artigo Original}

\section{Saúde Funcional}

11. Pacheco T, Luz SCT, Kock DB. Avaliação do equilíbrio antes e após a aplicação de um protocolo de tratamento com o Wii-Reabilitação: um estudo de caso com amputado de membro inferior. Artigos de periódico (graduação). Florianópolis: UFSC, 2013.

12. Vaghetti CAO, Botelho SSC. Ambientes virtuais de aprendizagem na educação física: uma revisão sobre a utilização de Exergames. Ciências e Cognição 2010; 15(1):76-88.

13. Schiavinato AM, Baldan C, Melatto L, Lima LS. Influência do Wii Fit no equilíbrio de paciente com disfunção cerebelar: estudo de caso. Health SciencesInstitute 2010; 28(1):50-2.

14. Rojas VG, Cancino EE, Silva CV, Lopéz MC, Arcos JF. Impacto delentrenamientodel balance através de realidade virtual em uma población de adultos mayores. InternartionalJournalMorphol 2010;28(1):303-8.

15. Lima NMFV, Rodrigues SY, Fillipo TM, De Oliveira R, Oberg TD, Cacho EWA. Versão brasileira da Escala de Comprometimento do Tronco: um estudo de validade em sujeitos pós-acidente vascular encefálico. Revista Fisioterapia e Pesquisa 2008; 15(3):248-53.

16. Alvarez MB, Fávero FM, De Sá CS. Avaliação do equilíbrio de pacientes com distrofia muscular de Duchenne. Revista Acta Fisiatra, 2011;18(2):49-54.

17. Carvalho EPS, Da Silva JB, De Alencar LL, De Jesus RLR, De Matos LKBL, Mesquita LSA, De Carvalho MEIM. Estabilometria e baropodometria na avaliação do equilíbrio em paciente com malformação de Arnold Chiari submetido a tratamento fisioterapêutico. Revista Terapia Manual 2013;11(53):355-60.

18. Shumway CA, Woollacott MH. Controle Motor: Teoria e aplicação prática. São Paulo: Manole, 2003.

19. Kelly CR, Redford JB, Zilber S, Madden PA. Standing balance in healthy boys and in children with Duchenne Muscular Dystrophy. ArchPhys MedRehabil1981;62(7):324-27.

20. Alfieri FM, Teodori RM, De Guirro RRJ. Estudo baropodométrico em idosos submetidos à intervenção fisioterapêutica. Revista Fisioterapia em Movimento 2006; 19(2):67-74. 


\section{Artigo Original}

\section{Saúde Funcional}

21. Barcala L, Colella F, Araujo MC, Salgado ASI, Oliveira C S. Análise do equilíbrio em pacientes hemiparéticos após o treino com o programa Wii Fit. Fisioterapia Movimento2011;24(2):337-43.

22. Joo LY, Tjan SY, Donald X, Ernest T, Chia PF, Christopher KKW, et al. A feasibility study using interactive commercial off-theshelf computer gaming in upper limb rehabilitation in patients after stroke. Journal Rehabilitation Medicine 2010;42:437- 41.

23. Castro FM. Estudo baropodométrica de pacientes com diabetes melitus tipo 2. (dissertação). Fortaleza: UNIFOR, 2007, 72p.

24. Aminian A, Sangeorzan BJ. The anatomy of cavus foot deformity. Foot Ankle Clin2008;13(2):191-8.

25. Dorneles PP. Análise do índice do arco plantar, equilíbrio postural e frequência do uso de salto altos em mulheres de diferentes faixas etárias (Tese). Porto Alegre: UFRGS, 2013, 79p.

26. Lafayette KCS, Mattos HM, Pacheco MTT. A influência podal na postura analisada através da baropodometria. Revista Univap 2005;12:1458-61. 